\title{
Real-time beam tracing for control of the deposition location of electron cyclotron waves
}

\author{
M. Reich, R. Bilato, U. Mszanowski, E. Poli, C. Rapson, J. Stober, F. Volpe, R. Zille \\ and ASDEX Upgrade team
}

\begin{abstract}
Plasma control techniques that use electron cyclotron (EC) resonance heating and current drive such as control of neoclassical tearing modes require accurate control of the deposition location of EC beams. ASDEX Upgrade has successfully implemented a real-time version of the beam-tracing code TORBEAM into its real-time diagnostic system to act as a globally available module that calculates current deposition location and its sensitivity from other real-time diagnostic measurements for all its moveable EC wave launchers. Based on a highly $(100 \mathrm{x})$ accelerated version of TORBEAM, the software implementation as a diagnostic process uses parallelization and achieves cycle times of 15-20 ms for determining the radial deposition location of 12 beams in the plasma. This cycle time includes data input-output overhead arising from the use of available realtime signals. The system is by design portable to other machines such as ITER.
\end{abstract}

Keywords: ECRH, ECCD, real-time, control of deposition location, beam tracing

\section{1) Introduction}

Real-time control of nuclear fusion plasmas is evolving from simple feedback-control of magnetic coils and single-input single-output feedback control of plasma density towards complex integrated control using multiple actuators with the ultimate goal of optimizing and eventually maintaining high plasma performance. Plasma control systems are starting to make use of multiple real-time measurements and calculate adequate response of the appropriate actuators [1]. One of the most flexible of the currently available actuators is electron cyclotron resonance heating (ECRH) and current drive (ECCD) which have been identified to be useful for a multitude of control tasks such as adjusting density peaking [2], sawtooth control [3,4,5], disruption avoidance [6] and neoclassical tearing mode (NTM) control [5,7,8 and references therein].

The paper addresses a software-layer for utilization of real-time controllable EC-wave launchers for the application of control of the ECCD deposition location to active MHD control. After the introduction and presentation of the challenge (section 2), the paper discusses the relevant code acceleration and validation (section 3). Its integration into the real-time framework of ASDEX Upgrade with the use of the Message Passing Interface (MPI, [9]) to benefit from modern multicore CPUs is the topic of section 4. Section 5 describes the implementation of the ECRH launcher control within the discharge control system (DCS). Section 6 finishes with the conclusions.

\subsection{Motivation}

Neoclassical Tearing Modes (NTMs) are regularly observed in high performance plasmas at reactorgrade $\beta$-values. They severely limit the maximal achievable normalized beta $\beta_{\mathrm{N}}$, which is undesirable because fusion performance scales as $\mathrm{P}_{\text {fusion }} \sim \beta_{\mathrm{N}}{ }^{2}$. NTMs may also lead to disruptions and thus need to be controlled. ASDEX Upgrade has been developing a feedback system for NTM stabilization, which uses real-time mode detection and localization [10]. For that, the ECRH deposition location needs to be accurately aligned with the NTM island. Initially, we followed the idea of measuring the ECCD deposition by modulating the power at frequencies compatible with the required timescales and calculating the deposition location with TORBEAM only as a crosscheck at lower time resolution, since the deposition location of a modulated ECRH beam is well measurable in low-confinement plasmas using modulation frequencies below $100 \mathrm{~Hz}$ with the $\Delta \mathrm{T}_{\mathrm{e}}$ modulation above the noise level of the observing ECE diagnostic. However, at higher modulation 
frequencies and in high power plasmas this could not be realized due to much lower $\Delta \mathrm{T}_{\mathrm{e}}$ amplitude and ECE diagnostic limitations. The need for an accurate and fast estimate of the ECCD deposition could thus only be satisfied by strongly accelerating the beam-tracing module.

While a potentially simpler system has been demonstrated that measures and actuates in the same reference frame, namely "ECCD based NTM stabilization using inline ECE" [11], that method also has disadvantages. Besides the intrinsic technical difficulties of separating $1 \mathrm{MW}$ of outgoing ECRH power from incoming ECE radiation (at below a few $\mathrm{nW}$ ), such a system needs to align its typically narrow plasma view with the NTM before it can operate. If a system needs prealignment with rational surfaces, where NTMs may develop, its availability as an actuator for other control tasks is limited at best. Moreoever, the inline ECE method is only suitable for active stabilization and cannot be used for preemptive ECCD on rational surfaces. To avoid such a limitation, we have chosen to adopt a more general strategy based on normalized coordinates which also allows to use the same controllers to execute other control tasks by feeding different deposition references as target locations. Since our chosen technique does not preclude the use of inline ECE, e.g. for finetuning the deposition location after pre-alignment, this method is also presently investigated at ASDEX Upgrade using the FADIS system [12].

\section{2) Boundary conditions}

Throughout the whole paper, coordinates expressed in normalized magnetic flux are based on the poloidal magnetic flux $\Psi$ in the following way:

$$
\rho_{\mathrm{pol}}=\sqrt{\left(\left(\Psi-\Psi_{\mathrm{a}}\right) /\left(\Psi_{\mathrm{s}}-\Psi_{\mathrm{a}}\right)\right)}
$$

where index s refers to the separatrix (where $\rho=1$ ) and index a to the magnetic axis (where $\rho=0$ ). 2.1 Code data input and output

TORBEAM [13] needs as inputs the magnetic equilibrium, density and temperature profile as well as a number of parameters describing the initial conditions of the ECRH beam. In order to meet our time-scale requirements, all input data need to be provided with least possible latency. Several realtime diagnostics provide the data to the real-time network, where the real-time TORBEAM process, also called a 'diagnostic' abbreviated as TBM, can access these data [14]. Our implementation makes the TBM process also the master of an MPI domain distributing workload across a number of other processes as required. The output of the real-time TORBEAM version consists of four values for the deposition location coincident with the peak power deposition within the plasma: normalized magnetic flux, radial, vertical and lateral (toroidal offset) coordinate. For receiving and sending real-time data, TBM uses the real-time framework [15] of ASDEX Upgrade and reflective memory as a transport layer for smallest possible latency between itself and the data sources and sinks.

\subsection{Why real-time?}

If there were no unforeseeable conditions that can change the behavior of our actuator we wouldn't need real-time calculations but could instead use prior knowledge (e.g. look-up tables) and run all actuators in a feed-forward mode. However, this is typically not the case. Experience shows that plasma conditions may change through normal but unwanted or abnormal events that may require immediate intervention for safe operation. When such events occur, too simple models are prone to failure: e.g. strong density peaking which leads to ECRH beam refraction, sawtooth crashes which change core transport on fast timescales, etc. As an indication for the large error that unforeseeable plasma effects can cause, a calculation using TORBEAM was performed, with two density profiles from the same discharge at different, yet not too distant time points $\mathrm{t}=3.1 \mathrm{~s}$ and $\mathrm{t}=3.5 \mathrm{~s}$. While all other plasma parameters and launching parameters are kept exactly the same, the peak deposition of ECRH power is different for the two cases by approximately $20 \mathrm{~cm}$ in vertical direction because of beam refraction (cf. figure 1) which equals more than 0.15 in normalized magnetic flux and corresponds to about $8 \mathrm{~cm}$ radial distance in the horizontal mid-plane. It is thus clear that by 
neglecting the density profile it is impossible to know the deposition location to better than several centimeters. While moderate changes in the density profile might still be possible to be prescribed by look-up tables, changes of other parameters such as plasma position, triangularity, etc. must also be accounted for.

Additionally, with respect to generic use of actuators, one piece of hardware (e.g. ECRH/ECCD) is desired for many applications: control of sawteeth, NTMs, density peaking. Thus, a controller that is designed to set launching parameters of the ECRH beam, e.g. by tilting a launching mirror, may be requested to switch between several tasks, each requiring heating power at a different location. Each of those control tasks may have higher or lower priority over the course of long plasma discharges, such that optimizing the controller for one or the other task leaves it less effective or even useless for others. In order to be prepared for all possible (also future, currently unknown) applications, it is necessary to ensure accurate control over the beam and its effect at the deposition location in any possible configuration, which usually depends on the plasma state.

As long as the number of necessary parameters to adequately describe the plasma state can be limited to less than 5, it would be feasible to generate a look-up table with up to 10 values in each dimension that will hopefully cover the full parameter space and hence provide a fast deposition calculation. However, a calculation of such a look-up matrix would already need about $10^{5}$ TORBEAM runs $(\approx 28 \mathrm{CPU}$ hours, assuming 1 s per run). If the number of necessary plasma and machine parameters exceeds 10 , even 7 values in each parameter dimension already cost about 60 CPU-years $\left(\approx 7^{11} \mathrm{~s}\right)$. Still, the real-time measurements of the parameters need to be taken and will cause some overhead which grows with size of the look-up table. Thus, in our opinion, it is not sensible to use the look-up table approach. A real-time capable calculation using a physically correct model is the best possible solution at present, especially since the required timescales for the real-time calculation will be less demanding in future larger devices. Moreover, such a solution is by design portable to other machines where the base (TORBEAM) is also operational.

\subsection{Time-scales}

The relevant timescales for NTM control are related to the current diffusion time (radial movement of the NTM) and the NTM growth time ( resistive timescale) and thus are on the order of $150 \mathrm{~ms}$ at ASDEX Upgrade. Since a rule-of-thumb for effective control requires about a factor of 10 oversampling in the control loop, we are aiming at $15 \mathrm{~ms}$ for the actuator. Strong acceleration of the code TORBEAM was necessary to achieve such cycle times. By meeting the cycle time target, successful controlled NTM stabilization could be demonstrated in several cases [16].

The discharge control system (DCS) at ASDEX Upgrade acts in every control cycle with a period of about $1 \mathrm{~ms}$. This time is comparable to the cycle time of the real-time equilibrium reconstruction code JANET [17] that produces magnetic quantities and is used as the basis for coordinate mapping. Density profiles from DCN interferometry [18] are generated with $1 \mathrm{~ms}$ cycles. Actuator control is done by the control system directly, such that the fast mirrors, which are capable of changing the ECRH beam direction with up to $12 \mathrm{deg} / 100 \mathrm{~ms}$, are addressed with least possible latency. The resulting change in deposition radius depends on plasma shape, density profile and horizontal position of the resonance layer of the incident wave, which is predominantly determined by the toroidal magnetic field. For ASDEX Upgrade parameters $\left(r_{\text {minor }}=0.5 \mathrm{~m}\right)$ the control system can thus change deposition by up to $15 \mathrm{~cm}$ in $50 \mathrm{~ms}$ when mapped to the radial distance in the midplane, the exact range depending on the plasma state and launcher parameters.

\subsection{Advantages and disadvantages}

A major advantage of the generic approach in comparison to highly specialized applications is the capability of adapting to previously unexpected situations. While a specialized controller has to have certain preconceptions built-in by design in order to meet a target performance, our scheme allows to properly treat also exceptional events and adequately treat new situations as long as those are described by the underlying model. It doesn't matter anymore which event changes the density profile as long as it is properly measured. By running different control tasks on the same type of 
actuator at different times during a long (reactor: steady-state) plasma discharge, sharing of the single actuator removes the need for installation of multiple specialized systems, each applicable to a different task. Priority decisions on which control task to favor at any given time can safely be left to other systems [19]. The generic model benefits further from its straightforward interface to other plasma-state observing systems such as RAPTOR [20] which can predict such out-of-order situations. A generic controller can identify the highest priority task and react accordingly, while specialized controllers might make the overall situation worse by operating only within their (limited) domain.

The disadvantages of the generic approach are that they rely on an accurate and fast equilibrium reconstruction. The equilibrium serves as the baseline reference coordinate system and is so important that many advanced control tasks would have to halt if there was a failure. Regarding the beam-tracing itself, not only the equilibrium data is needed but also accurate and fast information on the density (and to lower order temperature) profile as well as the machine parameters (launcher settings). If any of those are missing or are systematically wrong for some reason, the control of the deposition location and all dependent control mechanisms will fail.

In order to minimize disadvantages of the generic approach, all real-time diagnostics at ASDEX Upgrade apply a system of qualifiers to all measured or processed data. A diagnostic such as TBM that relies on several relevant inputs, most prominent of which are plasma equilibrium, density and the launcher state, needs to be aware of diagnostic failures which are communicated by attaching a quality tag to each sample. TBM forwards its higher level output with a new quality tag derived from all incoming signal tags and its own quality assessment of the produced results to DCS by tagging the results either GOOD or CORRUPT. In case of CORRUPT data being sent to the controller, any launcher movement is suspended until new GOOD data are received.

\section{3) Real-time TORBEAM}

The code TORBEAM solves the equations of the paraxial beam tracing method ([21] and ref. therein). It has been constantly benchmarked against other EC codes [22] and is routinely employed for heating, current drive and reflectometry applications at ASDEX Upgrade and other tokamaks, including ITER [23,24]. A call to the offline (i. e. not for real-time applications) version of TORBEAM encompasses three basic steps: (1) the input data are read from file and arranged on the required grids and some geometrical quantities (volumes, flux-surface averages) are computed; (2) the beam tracing equations, which build a set of ordinary differential equations describing the propagation and absorption of the wave beam, are integrated starting from the launch mirror until complete absorption (to numerical accuracy) is reached or until the beam leaves the plasma; (3) the absorption and current drive profiles are reconstructed from the data calculated during the integration (step 2). Thus, the standard TORBEAM output consists of "global" values (total absorbed power, total driven current) and of the radial profiles of the absorbed power density and driven current density. For real-time control of the deposition location, however, the integration of the beam path stops when the power absorbed at a given integration step is smaller than at the previous step and the four values mentioned in Sec. 2a (radial position and the three coordinates of the position of peak absorption) are returned. The position calculated in this way is used as an approximation to the position of the maximum of the absorption profile calculated employing the full beam structure. Moreover, it is assumed that the maximum of the absorption profile coincides with the maximum of the current profile in applications (such as NTM stabilization) where the latter is the relevant quantity. Both these assumptions have been checked by comparing the results of the accelerated and the complete version of the code and have been found to be satisfied within the limits required by real-time applications.

These considerations have allowed a first speed-up of the code by excluding from the calculations those parts which are not strictly necessary to obtain the reduced output described above. Since the geometrical quantities are not required for the integration, their evaluation is excluded from step (1). 
Moreover, the calculation of the beam cross section (beam width and curvature of the phase front, determined by 12 of the 19 beam tracing equations) and of EC current drive are excluded from step (2). Finally, step (3) is omitted entirely. The code acceleration due to the simplification of the physical model, as sketched above, leads to a reduction of the run time by a factor 20 from ca. $1.4 \mathrm{~s}$ to ca. $70 \mathrm{~ms}$. The detailed description of these modifications of the code and of further fine-tuning of the code parameters will be published elsewhere [25].

However, as mentioned in the introduction, a further speed-up by about a factor of four is required for effective control of ASDEX Upgrade discharges. A factor of three can be gained by properly setting the optimization options of the FORTRAN compiler used on the Sun architecture on which the workflow runs. Further changes in the code were more technical. With respect to its original version, the code has been transformed into a library, leaving to the calling process the input/output operations. The input parameters are passed to the library in form of 1-dimensional arrays to avoid conflicts or need for re-ordering (row-major vs. column-major) at the interface between the $\mathrm{C}$ (calling process) and FORTRAN (TORBEAM) parts of the workflow. In the case of multiple calls to the TORBEAM library for the same equilibrium, the dynamical allocation of the arrays is performed only once. Most importantly, the calculation of intermediate quantities (such as the radial coordinate) which is performed after each integration step, when the complete version of the code is employed, has been substituted by an extrapolation based on the values calculated at previous steps, the complete calculation being performed only at a preset frequency. These steps bring the execution time below the desired value of about $15 \mathrm{~ms}$ on the designated workstation.

The calculated deposition location of the real-time code only comprises the peak power deposition. Thus, as a validation test, we compare it thoroughly to the physically more accurate calculation, which returns both a full power and current deposition profile. The differences between peak power and peak current deposition are small, hence the real-time calculation skips calculation of current drive for saving CPU time. The final real-time result still has to meet certain accuracy requirements. To investigate this, 14 different plasma scenarios $\left(0.6 \mathrm{MA}<\mathrm{Ip}<1.0 \mathrm{MA},-3.1 \mathrm{~T}<\mathrm{B}_{\text {tor }}<-1.7 \mathrm{~T}\right.$, $2.210^{19}<\overline{\mathrm{n}}_{\mathrm{e}}<810^{19} \mathrm{~m}^{-3}$ ) with up to 60 timepoints distributed equally throughout each discharge have been selected to form a basic dataset for equilibrium data, density and temperature profiles. Within every dataset, one upper and one lower launcher have been scanned in poloidal ([-15, .., $+15])$ and toroidal $([-15, \ldots,+15])$ angle and both real-time code and offline version were run using the exact same input data, such that real-time deposition location, full version peak power deposition and peak current deposition could be determined with realistic input data over a large range of plasma and launcher configurations. The results for the deviation between real-time code and offline analysis are shown in figure 4 . While there is a small systematic trend near the magnetic axis, probably due to TORBEAM grid resolution, it is noted that the maximum deviation between real-time calculation and both peak power and peak current deposition does not exceed 0.03 in normalized poloidal flux anywhere. On average the absolute difference between current drive peak and real-time result in the range $0.1<\rho_{\text {pol }}<0.9$ is $2.610^{-3}$ with a standard deviation of $2.410^{-3}$ and the average mean being $310^{-4}$, which is strictly below the achievable accuracy when taking into account uncertainties in the input data. In fact, much larger uncertainties are produced by deviations in the real-time density profile.

For validating the code itself, a plasma experiment at low external heating power using ECRH on/off-modulation at $250 \mathrm{~Hz}$ was performed. While keeping the plasma conditions constant, the deposition location was changed in steps with plateaus lasting $0.5 \mathrm{~s}$ from near the magnetic axis to off axis using the poloidal steering capability of the launcher. In the chosen plasma, temperature fluctuations at the modulation frequency can readily be detected in ECE radiation temperature profiles, albeit only when using long enough integration time. An FFT analysis over $300 \mathrm{~ms}$ in each plateau was used to extract the fluctuation amplitude from ECE signals for each channel at the ECRH modulation frequency of $250 \mathrm{~Hz}$. The resulting profiles are plotted in figure 5 with the location of peak power deposition extracted from a Gaussian fit to those profiles. The fitted peak 
position and the corresponding calculation by TORBEAM are given in the following table:

$\begin{array}{ccc}\rho_{\text {pol }}[\mathrm{ECE}] & \rho_{\text {pol }}[\mathrm{TBM}] & \Delta \rho_{\text {pol }} \\ 0,221 & 0,228 & -0,007 \\ 0,379 & 0,358 & 0,021 \\ 0,555 & 0,528 & 0,027 \\ 0,693 & 0,681 & 0,012\end{array}$

The deviation of the TORBEAM calculation result from the experimentally determined deposition location does nowhere exceed 0.03 . Note that by using a comparatively low power plasma and near constant plasma conditions and by taking $300 \mathrm{~ms}$ of data for the evaluation of the deposition location, this dedicated experiment presents much simpler conditions for measuring the deposition location than typically present in the experiments, where the deposition needs to be known within $10-20 \mathrm{~ms}$ in plasmas with more heating, higher temperatures and thus much less distinguished modulation of the electron temperature.

\section{4) Implementation of TORBEAM in the discharge control system (DCS)}

Owing to the relatively complex formatting and necessary prevalidation of multiple data streams, real-time TORBEAM was implemented as part of a real-time diagnostic based on the existing realtime framework which already provides standardized exchange of real-time signals with DCS. As this real-time framework had been designed as a C-library containing an obligatory main routine and TORBEAM is a FORTRAN code, one of the early design decisions was to change TORBEAM into a library that can be called externally from all modern programming languages. This has conveniently also simplified modeling of the whole control loop [26] while using the exact same code as in real-time. Calls to this library allow the process TBM (by using real-time data received from other diagnostics through DCS) to act as the real-time TORBEAM module. Multiple parallel calls to the library with different starting parameters which tackle multiple actuators and generate derivative information is also possible, the details of which are discussed later.

The equilibrium data is received by TBM as an $m \times n$ matrix (where $\mathrm{m}$ and $\mathrm{n}$ are dynamically changeable parameters communicated through DCS before a discharge) with each matrix element representing the value of normalized magnetic flux at a grid point in the poloidal plasma crosssection. Each equally spaced grid point is assigned a radial coordinate $\mathrm{R}$ calculated from $\mathrm{R}_{\min }$ and $\mathrm{R}_{\max }$, which are additional parameters exchanged before the discharge. The vertical position $\mathrm{z}$ of each grid point is treated analogously. From that matrix and some complementary parameters, the TBM process first generates the equilibrium data vector for TORBEAM, which contains radial and vertical coordinates of the grid, values of $B_{\text {tor }}, B_{r}$ and $B_{z}$ and magnetic flux $\Psi$ at each grid point.

The density profile, the result of an inversion of line-integrated plasma density measurements, is based on the same equilibrium matrix as the one received by TBM and communicated as a polynomial expression with 4 coefficients. Therefore, the reconstruction of the profile with respect to normalized magnetic flux and subsequent generation of the appropriate density data is straightforward.

Since the temperature profile typically only affects the location of peak absorption by less than $1 \%$, we use an approximated profile derived from poloidal beta instead of a directly measured profile. This is both faster and we avoid numerical instabilities for cases where a measured profile might have artifacts and thus need special treatment. The profile (a typical H-mode profile with pedestal and constant core gradient) is generated using total plasma current, $\beta_{\mathrm{pol}}$ (volume averaged) and electron density at half-radius by the following formulas:

$$
\begin{aligned}
\mathrm{T}_{\max } & =\mathrm{C} \cdot \beta_{\mathrm{pol}} \cdot \mathrm{I}_{\mathrm{p}}^{2} \cdot\left[\mathrm{n}_{\mathrm{e}}(\rho=0.5)\right]^{-1} & & \mathrm{C}=3 \cdot 10^{8} \mathrm{keV} \mathrm{A}^{-2} \mathrm{~m}^{-3} \\
\mathrm{~T}\left(\rho_{\mathrm{pol}}\right) & =\mathrm{T}_{\max } \cdot\left(1-\rho_{\mathrm{pol}} / 1.2\right) & & \text { for } \rho_{\mathrm{pol}} \in[0 ; 0.9) \\
& =\mathrm{T}_{\max } \cdot\left(1.05-\rho_{\mathrm{pol}}\right) \cdot 5 / 3 & & \text { for } \rho_{\mathrm{pol}} \in[0.9 ; 1.05]
\end{aligned}
$$

Together with the density data, the resulting profile is used as the kinetic profile data vector. An additional real-time signal associated with the position of a push-rod that determines the 
launching mirror's alignment is used to calculate the parameters toroidal and poloidal angle. This calculation has to be done separately for each of the beams to get the respective machine parameters data vector.

When all incoming data (plasma equilibrium, density and temperature profiles and the poloidal and toroidal angles of the incident beam) qualify for use by having an acceptable confidence level, the minimal input data set for TORBEAM is completed with other required parameters such as desired integration accuracy and flags to switch optional calculation modes.

To optimize the gain factors, the global PID deposition location controller needs in addition to the current deposition location the steering sensitivity $\left(\mathrm{d} \rho_{\mathrm{dep}} / \mathrm{dX}\right)^{-1}$ at the current position which can be approximated using the first derivative of the functional dependence between deposition location $\left(\rho_{\text {dep }}\right)$ and actuator input signal $(X)$. Real-time TORBEAM therefore calculates the deposition location for any given input data set not only for the actual mirror state, but also for a second $(+\Delta \mathrm{X})$ and a third $(-\Delta X)$ setting, offset by a small step at the push rod controlling the mirror. In this way, a numerical approximation of the sensitivity can be obtained. In total, for a quadratic approximation of the sensitivity and $\mathrm{N}$ beams, $3 \mathrm{~N}$ calculations using almost identical input data are necessary at each time step. Since the memory footprint of the TORBEAM code even together with the complete input data for a single time-step is not an issue on modern computers, the necessary calculations can be done in parallel. The framework chosen for this parallelization was MPI [9].

While the TBM process does all the management tasks of collecting the real-time plasma data and converting it into the proper format, this step has to be done only once, such that the MPI setup was chosen to have a master process (TBM) and several slave processes (SLAVE) that only need to contain a bare minimum of code to do the calculations. The collection of results and further processing to produce all desired output is not relevant to the total cycle time (few tens of microseconds) and can be done solely by the master process. A schematic of the whole data transfer and task sharing with SLAVE processes is shown in figure 2.

Message passing

The MPI initialization is already built into the real-time diagnostic framework, such that only the slave processes need to be setup to match the initialization. This is achieved with the standard calls:
MPI Init (\&argc, \&argv);
MPI_Comm_size(MPI_COMM_WORLD, \&nproc);
MPI_Comm_rank(MPI_COMM_WORLD, \&myid);

While the master process TBM $\bar{a}^{-}$ssembles the complete set of input data, the slave processes just wait for the data to arrive. The data is distributed with a number of broadcasts and some sends. Since some processes are calculating only a specific configuration, some bandwidth is saved by omitting the distribution of all information to all slave processes (MPI_Bcast) and instead using MPI Send.

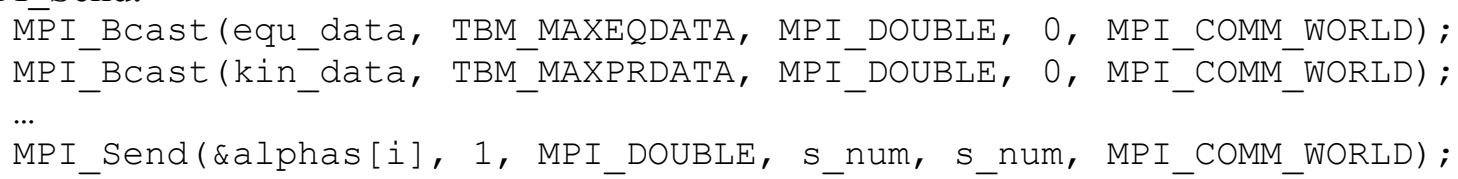

With the completion of data transfer, all processes (including the master process) calculate the single result they are configured to produce. Finally the results are combined by doing:

MPI_Gather(res, 1, MPI_DOUBLE, rho, 1, MPI_DOUBLE, 0, MPI_COMM_WORLD) ;

While the slave processes at this point return to a waiting state, the master process combines all individual results, calculates the sensitivity approximations and - using the same signal exchange mechanisms as used for acquiring the input data set - sends the results to DCS, where the appropriate controller then uses it for controlling the actuators according to the experiment needs.

The total integration time for a single beam trace is approximately proportional to the path length traveled until a maximum in power absorption is reached. For some cases where the cold resonance layer lies far on the high field side or for a plasma with a strong density gradient where due to strong refraction of the beam absorption is not at all achieved inside the plasma, the consequence 
may be a longer execution time. On the currently used workstation, an x 86 computer with 24 virtual cores at $3.4 \mathrm{GHz}$ running Solaris 10, the whole calculation takes between $10 \mathrm{~ms}$ and $30 \mathrm{~ms}$. With this setup, TBM delivers control signals for all 4 controllable launchers, calculating 12 beams in parallel representing the central and 2 peripheral beams for 4 individual launchers. The calculation times depend strongly on the radial position of the absorption region. For our most common scenario with the vertical resonance layer for $\mathrm{X} 2$ mode through the plasma center $\left(\mathrm{B}_{\text {tor, axis }}=2.5 \mathrm{~T}\right.$, $\mathrm{v}=140 \mathrm{GHz}$ ) and typical density profiles are on average $12 \mathrm{~ms}$ on the dedicated workstation, which satisfies our requirements ( $<15 \mathrm{~ms}$, even with an extra safety margin of $\approx 20 \%$ ).

\section{5) DCS launcher control}

A feedback controller is used to steer the launcher mirrors such that the deposition location $\rho_{\text {dep }}$ can track a given reference $\rho_{\text {ref. }}$ References can come from the equilibrium (e.g. $\rho_{\mathrm{q}=1.5}, \rho_{\mathrm{q}=2.0}$ ) or from the correlation of ECE and Mirnov measurements (e.g. $\rho_{\mathrm{n}=1}, \rho_{\mathrm{n}=2}$ ) or as defined in the pulse schedule $\rho_{\text {Ps. }}$ The controller smoothly transitions from one reference to the other. Whichever reference is currently active, the controller calculates the error in $\rho\left(\rho_{\text {ref }}-\rho_{\text {dep }}\right)$ and multiplies it by the sensitivity $\left(d \rho_{\text {dep }} / \mathrm{dX}\right)^{-1}$. This performs gain scheduling using a linear approximation to the true non-linear and time-varying sensitivity and performs a coordinate transformation from $\rho$ to the units of the linear mirror actuator - i.e. meters. The result is fed into a standard PI (proportional-integral) controller, which was tuned using the offline version of rtTORBEAM and archived data [26]. During the tuning process, it was noted that TORBEAM does not filter sample-to-sample noise in the density profile or the equilibrium and thus produces some unavoidable jitter. In order to provide smooth trajectories to the mirror and avoid cyclic forces on the launcher, the control bandwidth is limited.

Two further precautions are taken to protect the launcher. Firstly, if TBM tags its result as CORRUPT (cf. section 2) the controller will keep the mirror stationary until it receives both valid $\rho_{\text {dep }}$ and $d \rho_{\text {dep }} / d X$. Secondly, when the deposition lies on flux surfaces close to the midplane, which are tangential to the ECRH resonance layer (see figure 3), it holds that $d \rho_{\text {dep }} / d X \approx 0$ and the sensitivity goes to infinity. Since this would result in large actuator commands, the sensitivity is interpolated between $d \rho_{\text {dep }} / d X=-10$ and $d \rho_{\text {dep }} / d X=+10$, i.e.

$$
\begin{array}{ll}
\text { Sensitivity }=\left(d \rho_{\text {dep }} / d X\right)^{-1} & \text { for }\left|d \rho_{\text {dep }} / d X\right|>10 \\
\text { Sensitivity }=0.01 \cdot\left(d \rho_{\text {dep }} / d X\right) & \text { for }\left|d \rho_{\text {dep }} / d X\right|<10
\end{array}
$$

This gives a smooth transition and limited gains when deposition is close to the midplane.

Apart from tracking references in $\rho$, the deposition location from TBM can be used to help detect unsafe use of ECRH. There is potential for TBM to detect situations such as unabsorbed power, beams that are reflected at cut-off, strongly refracted or simply aimed poorly. First attempts in that regard have been performed on ASDEX Upgrade, but routine use will have to wait until suitable limits for acceptable stray radiation can be agreed upon.

\section{6) Conclusions}

In order to address the issues arising with real-time control of neoclassical tearing modes, a fast and precise steering of ECCD deposition was needed. To this end, high acceleration of the TORBEAM code by a factor of approximately 100 and its integration with the ASDEX Upgrade real-time framework was performed. MPI was successfully used to keep the amount of low-level programming to a minimum, yet still achieve good performance by parallelization. The achieved result allows to run the real-time diagnostic in support of controlling the deposition of multiple ECwave launchers on a timescale under $20 \mathrm{~ms}$ without compromising the generality of the ray-tracing calculations, such that the diagnostic can be used for many different control goals across a wide range of plasma scenarios.

\section{Acknowledgements}


This project has received funding from the EURATOM research and training programme 2014-18.

\section{References:}

[1] W. Treutterer et al., "Integrated operation of diagnostic and control systems", Fus. Eng. Des., 86, 6-8, (2011), Pages 465-470, doi:10.1016/j.fusengdes.2010.12.074

[2] A. Mlynek et al., "Real-time feedback control of the plasma density profile on ASDEX Upgrade", Nucl. Fusion 51, 4 (2011), 043002, doi:10.1088/0029-5515/51/4/043002

[3] I T Chapman, "Controlling sawtooth oscillations in tokamak plasmas", Plasma Phys. Contr. Fus. 53 (2011) 013001, doi:10.1088/0741-3335/53/1/013001

[4] I T Chapman et al., "Sawtooth control using ECCD in ITER demonstration plasmas in DIII-D", Nucl. Fusion 52 (2012) 063006 doi:10.1088/0029-5515/52/6/063006

[5] F. Felici et al., "Real-time control of multiple MHD instabilities on TCV by ECRH/ECCD”, EPJ Web of Conferences 3202005 (2012), doi: 10.1051/epjconf/20123202005

[6] B. Esposito et al., "Disruption Avoidance in the Frascati Tokamak Upgrade by ... " Phys. Rev. Lett. 100 (2008), 045006, doi:10.1103/PhysRevLett.100.045006

[7] E. Kolemen et al. "State-of-the-Art NTM Control in DIII-D Using Real-time Steerable ECCD Launchers", Nucl. Fusion 54 (2014) 073020, doi:10.1088/0029-5515/54/7/073020

[8] M. Maraschek, "Control of neoclassical tearing modes", Nucl. Fus. 52, 7 (2012) 074007, doi:10.1088/0029-5515/52/7/074007

[9] http://www.open-mpi.org/

[10] M. Reich et al., "NTM localization by correlation of Te and dB/dt", Fusion Science and Technology 61 (2012), 309

[11]] E. Westerhof et al., "Tearing mode stabilization by electron cyclotron resonance heating demonstrated in the TEXTOR tokamak and the implication for ITER ", Nucl. Fusion 47, 3 (2007) 85, doi:10.1088/0029-5515/47/2/003

[12] Walter Kasparek et al., "Development of resonant Diplexers - Status, Applications, Plans", EC-18, Nara, Japan, 2014

[13] E. Poli et al., "TORBEAM, A Beam Tracing Code for Electron-Cyclotron Waves in Tokamak Plasmas", Comput. Phys. Commun., 136, 90 (2001); doi:10.10160S0010-4655(01)00146-1

[14] W. Treutterer et al., "Real-time signal communication between diagnostic and control in ASDEX Upgrade", Fus. Eng. Des., 85, 3-4, (2010), 466, doi:10.1016/j.fusengdes.2010.04.031

[15] M. Reich et al., "Real-time diagnostics and their applications at ASDEX Upgrade", Fusion Science and Technology 58, 3 (2010), 727.

[16] M. Reich et al., "Real-time Control of NTMs Using ECCD at ASDEX Upgrade", IAEA Fusion Energy Conference 2014, PPC/P1-26

[17] L. Giannone et al., "A data acquisition system for real-time magnetic equilibrium reconstruction on ASDEX Upgrade and its application to NTM stabilization experiments", Fus. Eng. Des. 88, 12 (2013), 3299-3311, doi:10.1016/j.fusengdes.2013.10.008

[18] A. Mlynek et al., "Real-time feedback control of the plasma density profile on ASDEX Upgrade ”, 2011 Nucl. Fusion 51 (2011) 043002, doi:10.1088/0029-5515/51/4/043002

[19] C. J. Rapson et al., "Actuator management for ECRH at ASDEX Upgrade", submitted to SOFT2014, Fus. Eng. Des.

[20] F. Felici et al., "Real-time physics-model-based simulation of the current density profile in tokamak plasmas" Nucl. Fusion 51 (2011) 083052, doi:10.1088/0029-5515/51/8/083052

[21] E. Poli et al., "EC beam tracing in fusion plasmas", Fus. Eng. Des. 53 (2001) 9, doi:10.1016/S0920-3796(00)00471-3

[22] R. Prater et al., "Benchmarking of codes for EC heating and EC current drive under ITER conditions", Nucl. Fusion 48 (2008) 035006, doi:10.1088/0029-5515/48/3/035006

[23] M. A. Henderson et al., "EU developments of the ITER ECRH system", Fus. Eng. Des. 82 (2007) 454, doi:10.1016/j.fusengdes.2007.01.029

[24] A. Stegmeir et al., "Analysis of the ITER low field side reflectometer employing the Beam 
Tracing Method", Fus. Eng. Des. 86 (2011) 2928, doi:10.1016/j.fusengdes.2011.07.006 [25] F. Volpe et al., Computer Physics Communications (2014), in preparation.

[26] Rapson, C. et al., "Simulation of feedback control system for NTM stabilisation in ASDEX Upgrade", Fus. Eng. Des., 2013, 88, 1137, doi:10.1016/j.fusengdes.2013.02.127 


\section{Figure captions:}

Figure 1: Illustration of systematic deviation of deposition for different density profiles but identical equilibrium data and launching parameters

Figure 2: Schematic interconnection diagram of participating nodes of the real-time TORBEAM process (EQR: real-time equilibrium, DCR real-time $\mathrm{DCN}$ density profile, $\alpha, \beta$ : launcher parameters)

Figure 3: Sensitivity is high when deposition is on a flux surface tangential to the resonance layer because deposition changes are miniscule with a comparatively large change of poloidal angle.

Figure 4: Deviation (for $>13000$ data points) between real-time code result and full version peak power and peak current deposition (CD) for selected datasets ranging over multiple plasma scenarios (discharges ranging from \#31000 - \#31400) in units of normalized poloidal magnetic flux across the whole plasma radius

Figure 5: Four different launching directions within the same discharge (\#26388) and the corresponding ECE temperature fluctuation profiles at the ECRH modulation frequency versus normalized radius with a gaussfit to each profile 

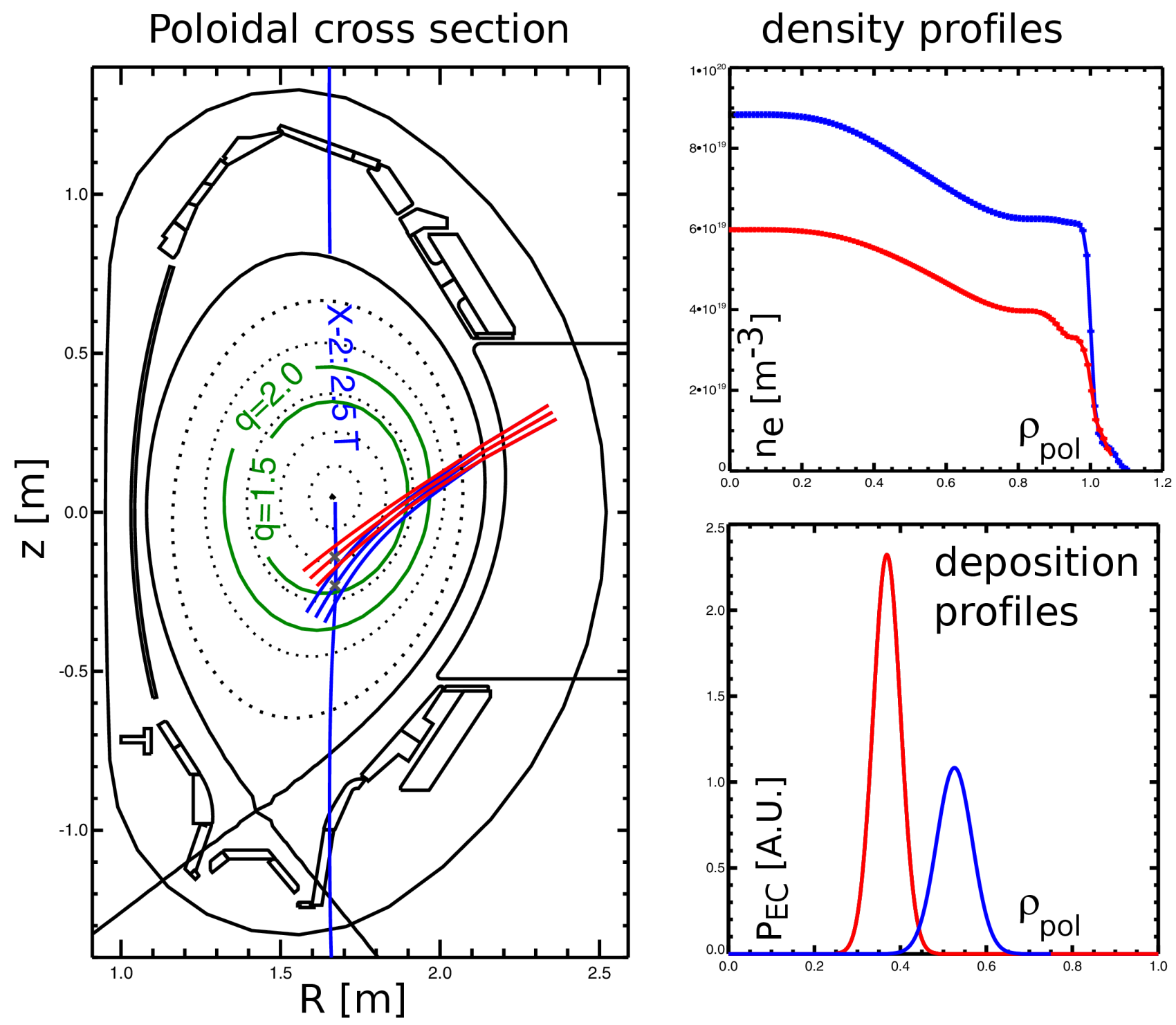

Figure 1: Illustration of systematic deviation of deposition for different density profiles but identical equilibrium data and launching parameters 


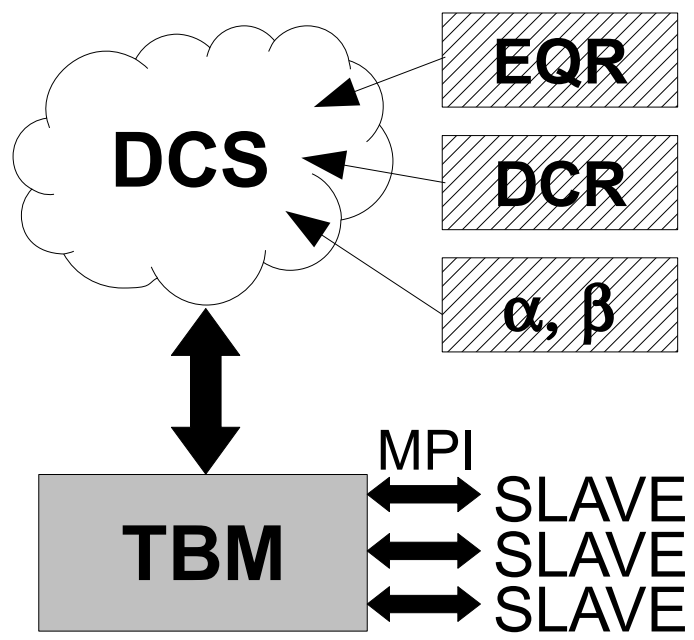

Figure 2: Schematic interconnection diagram of participating nodes of the real-time TORBEAM process (EQR: real-time equilibrium, DCR realtime $D C N$ density profile, $\alpha$, $\beta$ : launcher parameters) 


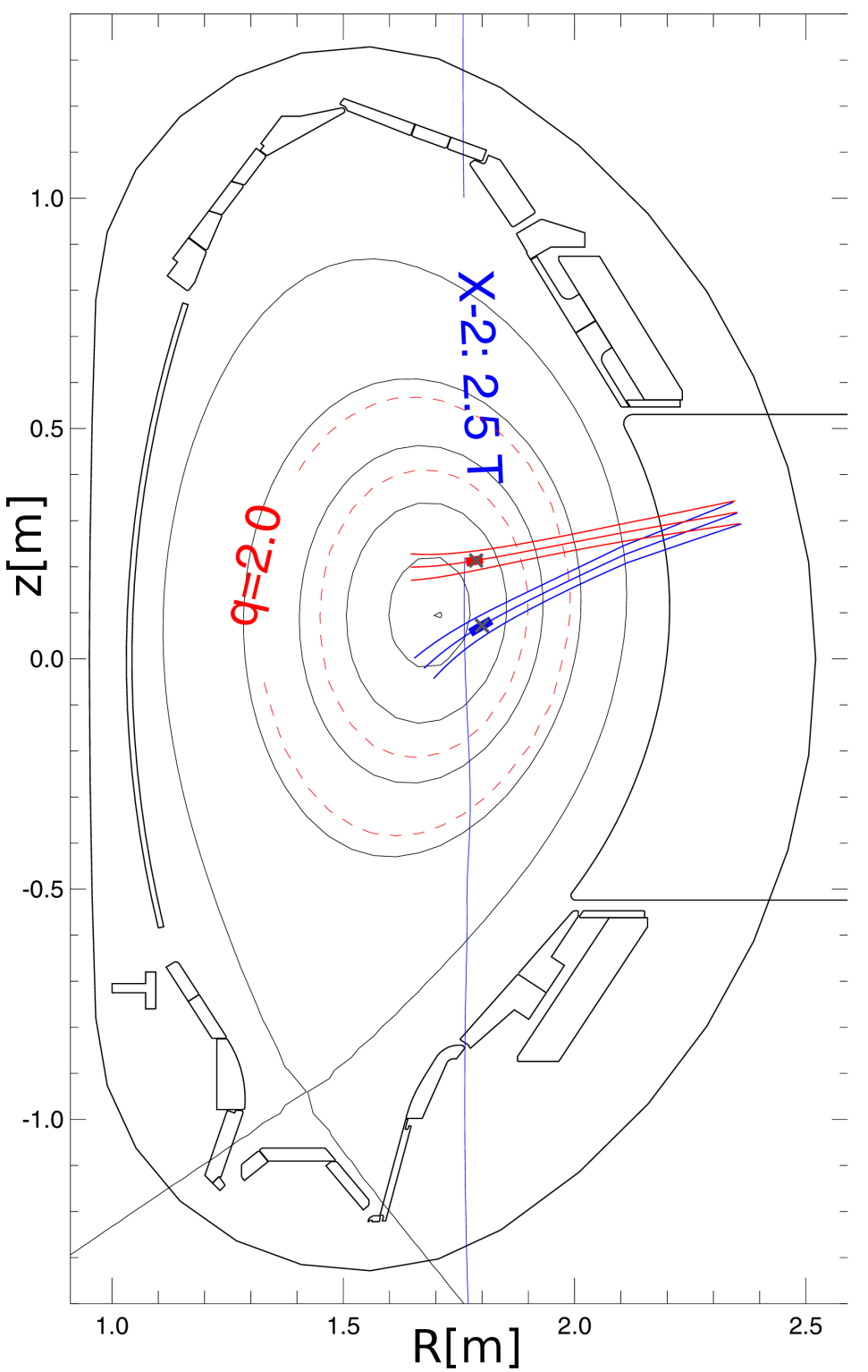

Figure 3: Sensitivity is high when deposition is on a flux surface tangential to the resonance layer because deposition changes are miniscule with a comparatively large change of poloidal angle. 


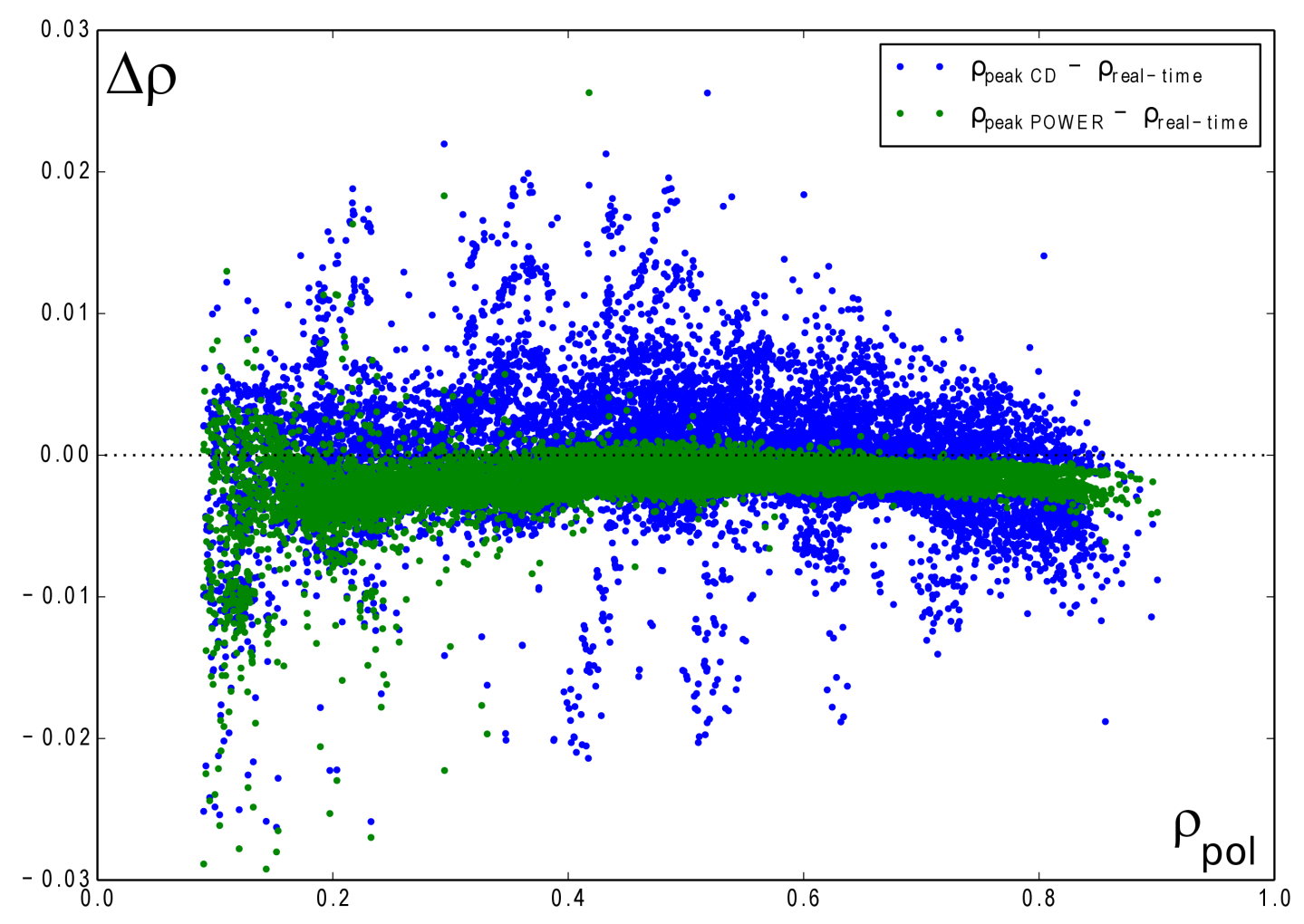

Figure 4: Deviation (for $>13000$ data) between real-time code result and full version peak power and peak current deposition (CD) for selected datasets ranging over multiple plasma scenarios (discharges ranging from \#31000 - \#31400) in units of normalized poloidal flux across the whole plasma radius. The small systematic offset is probably caused by the stop condition, which differes slightly in the real-time version. 


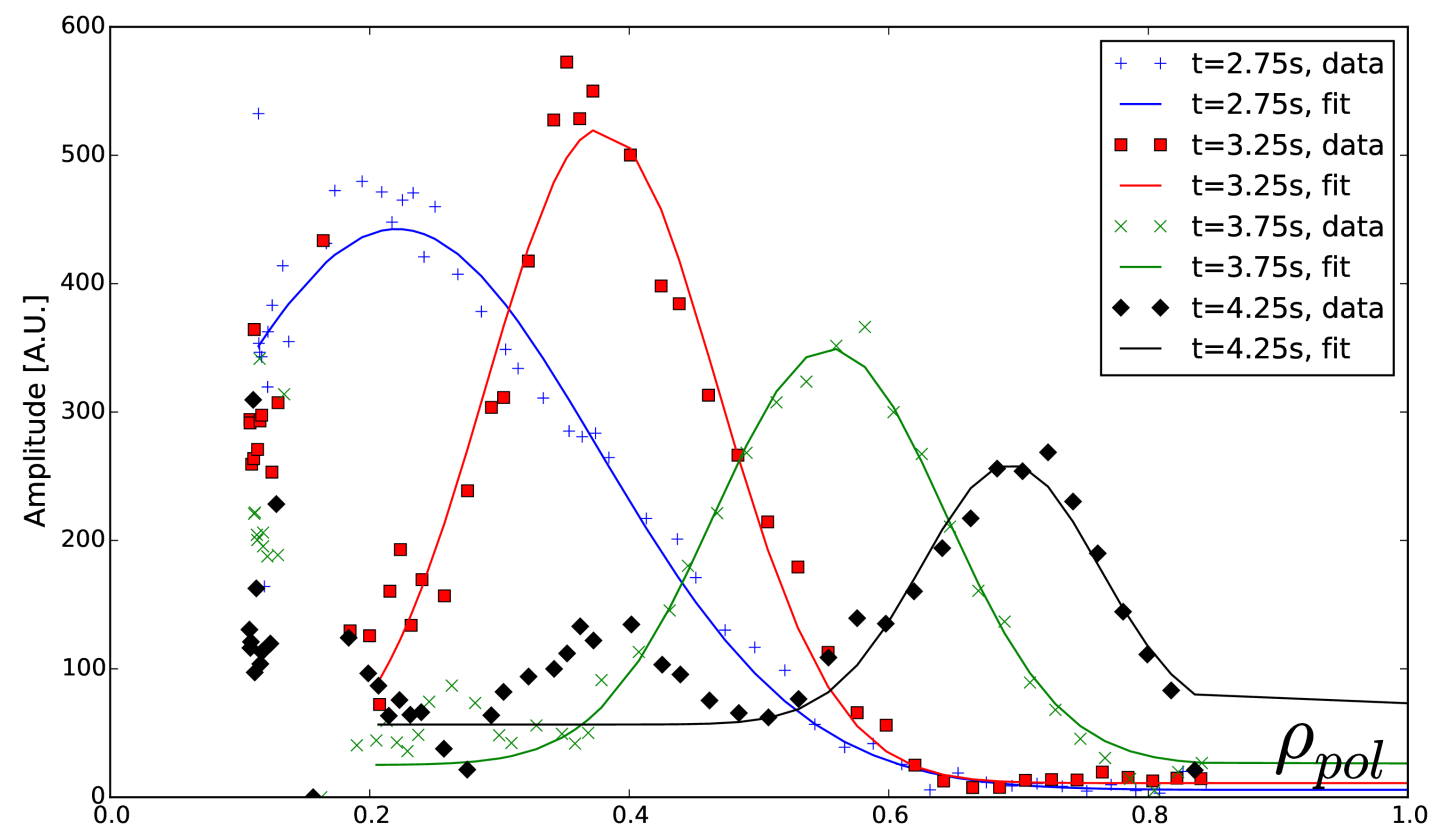

Figure 5: Four different launching directions within the same discharge (\#26388) and the corresponding ECE temperature fluctuation profiles at the ECRH modulation frequency versus normalized polidal flux with a gaussfit to each profile 